\title{
Assessment of the Ecological Niche of Photovoltaic Agriculture in China
}

\author{
Lingjun Wang ${ }^{1,2}$, Ying Wang ${ }^{1}$ and Jian Chen ${ }^{1,3, *(1)}$ \\ 1 College of Economics and Management, Nanjing University of Aeronautics and Astronautics, \\ Nanjing 211106, China; yingwang@nuaa.edu.cn (L.W.); yingwang@nuaa.edu.cn (Y.W.) \\ 2 Nanjing University of Science \& Technology Zijin College, Nanjing 210023, China \\ 3 College of Economics and Management, Nanjing Forestry University, Nanjing 210037, China \\ * Correspondence: fina@njfu.edu.cn; Tel.: +86-025-8542-7376
}

Received: 19 March 2019; Accepted: 12 April 2019; Published: 15 April 2019

\begin{abstract}
To evaluate the ecological niche of photovoltaic agriculture in China, an evaluation index system was constructed. Based on the presentation form of interval numbers, we used the interval entropy weight method and interval cloud model to measure the niche state value and niche role value of photovoltaic agriculture. In this way, we determined the development trend of the ecological niche of photovoltaic agriculture. The results show that Chinese photovoltaic agriculture is in a good state and plays a good, but weak, role. The ecological niche of China's photovoltaic agriculture will undergo a four-stage evolution process: positioning, integration, leap, and symbiosis. China has completed the positioning stage and entered the integration stage. Hence, it is important to constantly improve the level of industrial integration technology and to form a new photovoltaic agriculture recycling economic ecosystem.
\end{abstract}

Keywords: photovoltaic agriculture; ecological niche; interval cloud model; interval entropy weight method

\section{Introduction}

Photovoltaic agriculture is an innovative ecosystem that results from closely connecting photovoltaic power generation with modern agriculture [1]. It is a new idea that combines agriculture with photovoltaics in an organic way and is one of ways to transform from a simple industry to industrial integration [1]. As a form of land use and a direction for new development of photovoltaic power generation, photovoltaic agriculture is an outcome of integrating modern agriculture and green energy. It can make use of the zero-pollution advantage of photovoltaics to generate power while offering appropriate environmental support for agricultural products. On the one hand, it can facilitate the greening of agricultural production. On the other hand, it can save cost by using photovoltaic power generation to form low-carbon and cyclic ecological agriculture and achieve a win-win for economic and social benefits [2]. This system combines agricultural production and photovoltaic power generation under the condition that land properties remain unchanged. Hence, it plays a positive role in improving the comprehensive land use rate, developing modern effective agriculture, and increasing farmers' income. As an innovative mode of development, photovoltaic agriculture can promote the development of the photovoltaic industry. In particular, as China is making great efforts to improve the utilization rate of solar power, photovoltaic agriculture has grown rapidly in recent years and there are broad prospects for it [3,4]. In 2009, the installed capacity of China's photovoltaic agricultural power stations was less than $0.001 \mathrm{GW}$, but it reached $1.18 \mathrm{GW}$ in 2014. According to Frost and Sullivan, the annual installed capacity of China's agricultural photovoltaic power stations reached $3.26 \mathrm{GW}$ in 2018, and the cumulative installed capacity reached $12.42 \mathrm{GW}$. 
An ecological niche refers to the position of certain species in multidimensional space that is formed with environmental resources or conditions as gradients [5]. It is a key concept in ecology. With the rapid development of the economy and improvement of technological innovation, the niche theory is widely applied in the field of natural science and has been introduced into studies of social science. There is a lot of niche research on economics [6-8] and management [9-11], and these studies provide new ideas for economic and managerial decisions of social departments or related organizations. In particular, some scholars extend the niche theory, putting forward that an ecological niche includes two aspects, state, and role [12]. "State" refers to the current state and is the result of the learning and development of biology units in the past and the interaction with the environment, and "role" means the actual influence or power of biology units on the environment [13]. The two aspects of ecological niche can comprehensively reflect the accumulated results and future development trends of the past ecological action of systems, such as in enterprise or industry, and provide a new perspective for the study and evaluation of the ecological niche of these systems [14,15]. We construct an index system from the two aspects in Section 2.

The ecological niche of photovoltaic agriculture refers to the position in time and space, and the relationship and function within the system or with other industries. In the current situation of resource shortages and environmental pollution, how to make photovoltaic agriculture play an important role and create a win-win situation for the economy, society, and ecology is a problem that needs our attention. New technologies are "hopeful monstrosities" [16], and an ecological niche should be created to protect the technology's trajectory from the selection process [17]. So, technology or innovation systems require niche support to function and produce benefits [6]. In agricultural development settings, a niche also serves as a "protective space" [18], and well-designed innovation niches may facilitate transitions toward a sustainable agricultural future [19]. Hence, it is important to measure and describe the niche of photovoltaic agriculture and take steps to achieve a high-level ecological niche.

In the literature, there have been extensive and in-depth studies about the technology [20-22], prospects [1,23], and economic performance [3,24-26] of photovoltaic agriculture and the application of niche theory in areas of economy and management. However, there is a lack of research on the niche of photovoltaic agriculture. Without understanding it, we will not be able to manage this niche and improve its level. Therefore, based on niche theory, we evaluated the ecological niche of photovoltaic agriculture and put forward some suggestions to improve the level of its application.

The contributions of this paper are as follows: (1) Factors that affect the ecological niche of photovoltaic agriculture are identified, and an index system for measuring it is constructed from two aspects, state, and role. (2) Using a method based on the interval cloud model, the state value, role value, and integrated value of the ecological niche of Chinese photovoltaic agriculture is calculated, and then we determine its development stage. (3) Based on the calculation results, suggestions for promoting photovoltaic agriculture to develop a high-level niche are proposed.

The rest of the paper is organized as follows. The following section constructs the index system for measuring the state and role values of the ecological niche of photovoltaic agriculture. In Section 3 , an evaluation method based on the interval cloud model is presented. Section 4 summarizes the results and Section 5 discusses them. Our conclusions and recommendations are given in the final section of the paper.

\section{Construction of Index System for Evaluating Ecological Niche of Photovoltaic Agriculture}

\subsection{Analytical Framework}

To evaluate the ecological niche of photovoltaic agriculture, it is necessary to construct an index system. As mentioned in Section 1, the index system is constructed from the aspect of both the state and role of this niche. In addition, we further determine its development stage. The specific analytical framework is shown in Figure 1. 


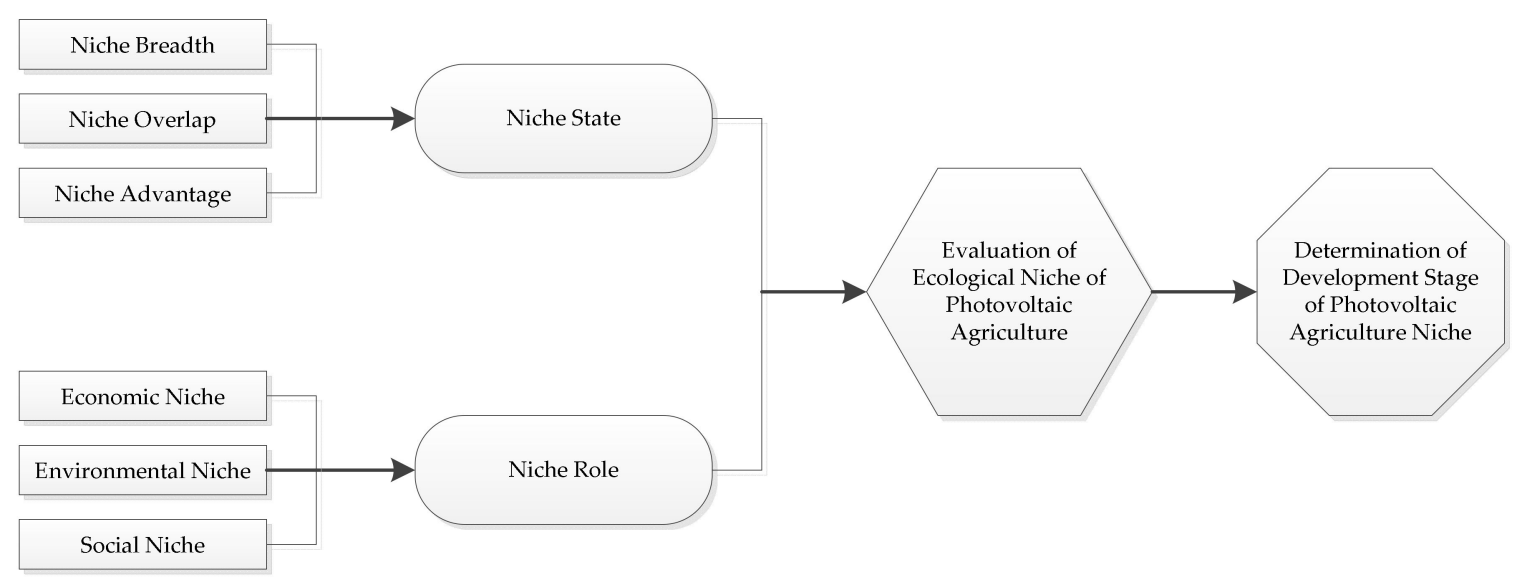

Figure 1. Analytical framework.

\subsection{Index System Construction}

According to the connotations of the state and role of ecological niche, the state of photovoltaic agriculture is an outcome of accumulated mutual effects between photovoltaic agriculture and the environment in terms of its past growth, study, and social and economic development. To sum up, the internal state and development level of photovoltaic agriculture present the self-accumulation state from its formation to the present, including three criterion-layer indices: niche breadth, niche overlap, and niche advantage [27]. Niche breadth refers to the clustering degree of resources especially for supporting certain species or the professional degree of resources being applied in certain species [28]. Niche overlap refers to the degree of several species sharing certain resources. When resources are limited, the number of overlapping dimensions will be higher, and the competition will be fiercer [29]. Niche advantage refers to the advantages of resource sharing, cost reduction, and policy support in the integration of two systems.

The niche role of photovoltaic agriculture refers to its actual impact on the external environment in the process of constant development. It is also the manipulating force that maintains sustainable and stable development of the system and the exchange of materials, energy, and information between the system and the external environment after two industries are integrated. The niche role of photovoltaic agriculture emphasizes the interaction between photovoltaic agriculture and the external environment, including three criterion-layer indices: economic niche, environmental niche, and social niche. The economic niche is the influence of the system on the external economic environment. In a photovoltaic agriculture system, the main manifestations are the economic benefits of electricity generation, crops, and other industries, such as tourism, and even the impact on the entire GDP. The environmental niche is the ability of the system to have a realistic impact on the external environment. In the system, it is mainly reflected in the reduction of external emissions of pollutants, such as $\mathrm{CO}_{2}$ and $\mathrm{SO}_{2}$. The social-ecological niche is the adjustment of the system to the social structure. The system mainly involves the adjustment of the power supply and demand structure and agricultural structure. The niche state and niche role of the system are not isolated but interact and complement each other. It is the mutual promotion of the niche state and niche role of photovoltaic agriculture that enables the continuous development of the system, steady steps to the next process, and realization of the high-level ecological niche.

This study makes a theoretical analysis of measurement indices of the ecological niche of photovoltaic agriculture, by following commenting rules, such as being comprehensive and objective, scientific, and practical, integrating qualitative and quantitative features, and being attainable and operable, and dynamic and oriented. After visiting and investigating more than 10 photovoltaic agriculture projects, such as Jiangsu Xiexin (Suzhou Xiexin Yongqiao project) and the Chinese Energy Construction Group's Yuyao agricultural tourism demonstration park project, and combining the state 
and role of the ecological niche of photovoltaic agriculture, we preliminarily selected 60 indices and constructed a preliminary index system to evaluate the ecological niche of photovoltaic agriculture. After that, an expert investigation method, relevance analysis, and other methods were applied to verify and select these indices. We also made field investigations and conducted direct interviews with 20 industry experts (primarily principle persons responsible for photovoltaic agriculture projects and experts studying photovoltaic agriculture) for their opinions. The experts have been in this field for a long time. We asked all of the experts to rate the importance of various indices according to their professional knowledge and practical experience. The total score was 50 (10 points: very weak, 20 points: quite weak, 30 points: general, 40 points: quite strong, 50 points: very strong). In total, 20 questionnaires were given out for the investigation, and 18 were collected. Finally, 17 effective questionnaires were attained. After scoring by experts for two rounds, index-level indices with average scores not higher than 30 were deleted and a photovoltaic agriculture niche evaluation index system was established with six criterion-layer indices $\left(S_{1}, S_{2}, S_{3}, R_{1}, R_{2}, R_{3}\right)$ and 24 index-level indices $\left(S_{11}\right.$, $\left.\ldots, S_{14}, S_{21}, \ldots, S_{24}, S_{31}, \ldots, S_{34}, R_{11}, \ldots, R_{14}, R_{21}, \ldots, R_{24}, R_{31}, \ldots, R_{34}\right)$, as shown in Figure 2.

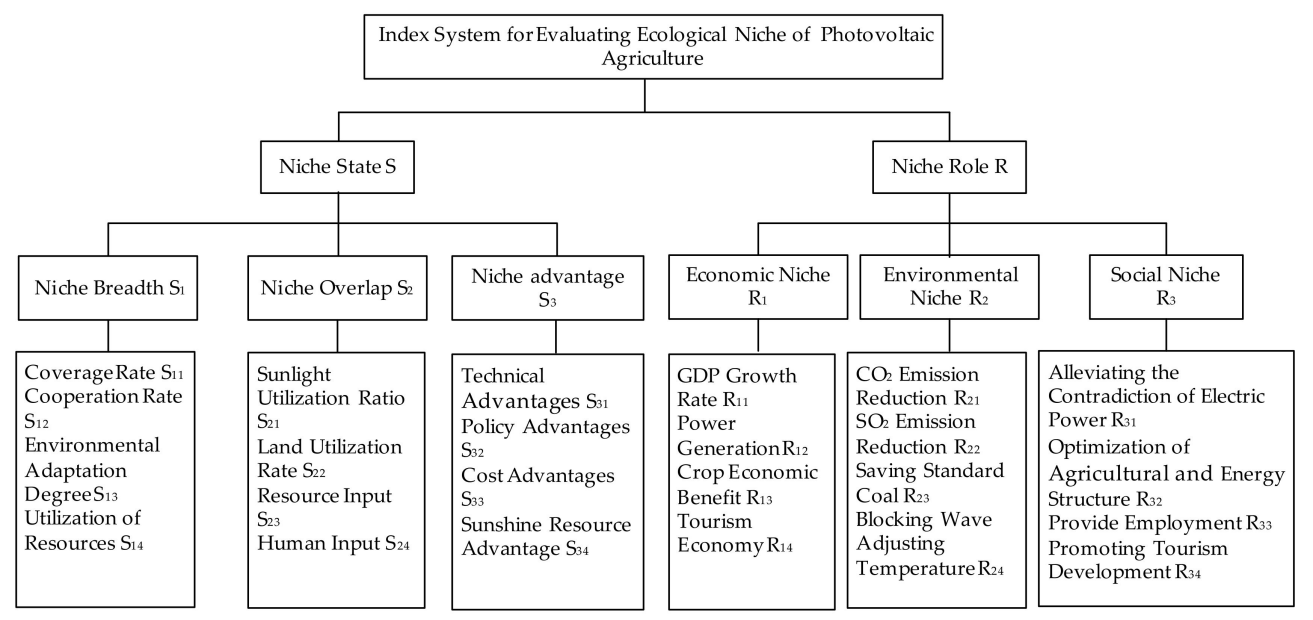

Figure 2. Index system for evaluating ecological niche of photovoltaic agriculture.

\subsection{Index Comment Set}

We established a set of comments on the evaluation indices: $\mathrm{V}=$ very weak, quite weak, general, quite strong, very strong. We invited experts in the industry to give interval values to the set of comments between 0 and 1 . Then we calculated interval expectation, entropy, and super entropy of every class of niche state value and niche role value. We used Matlab 9.1 to convert interval feature values of the comment set to an interval cloud picture, as shown in Figure 3.

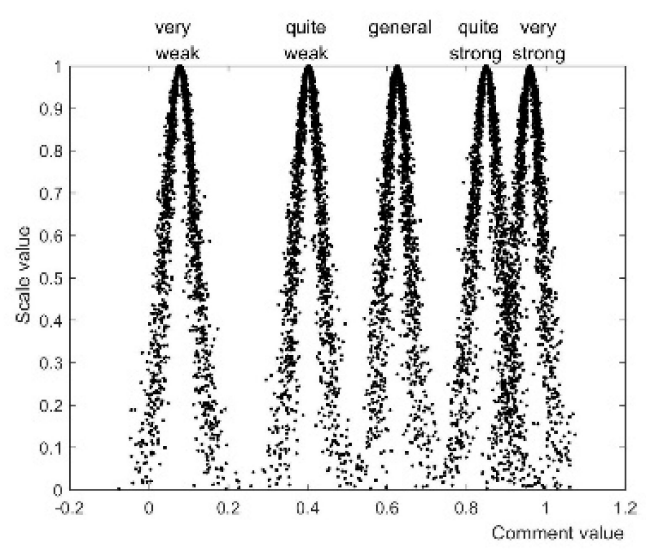

Figure 3. Cloud chart of comment set of ecological niche of photovoltaic agriculture. 


\section{Methods}

Due to the complexity and uncertainty of index weight and rating in the above evaluation index system as well as limitations of human thinking, common random process distribution functions and abstract functions in statistical subordinate functions of fuzzy mathematics are often not easy to determine when it comes to the uncertainty of ecological niche [30]. Compared with monotropic data, interval data are more conducive to presenting the valuing situation of datasets and revealing the overall features of data values [31]. Using interval numbers for analysis makes it get closer to the complicated but uncertain final assessment result as well as human thinking habits [32]. Hence, this paper uses interval numbers for index weights and values.

\subsection{Weight Determination}

First, the weight of the index is calculated. The entropy weight method is often used to calculate objective weight and widely applied in many areas, such as engineering projects, enterprise management, credit risk assessment, and financial property allocation [33-35]. First, calculate the index dispersion degree, find the index weight information from the original data, and calculate the weight of every index. The weight vector can be measured with a different degree among index data, so it features a high degree of objectivity [36-38]. Despite that, the original data for calculation indices are often uncertain. In particular, when index values are determined with the expert scoring method, it will look too arbitrary to describe qualitative indices with certain numbers. Hence, the interval algorithm and entropy weight methods are mixed together and the interval entropy weight method is put forward. The process for calculating the weight of every index is shown as the following steps:

Step 1: Establish a decision matrix.

Assume an index set $Q=\left\{Q_{1}, Q_{2}, \ldots Q_{m}\right\}$ and get a set of measurement objects $S=\left\{S_{1}, S_{2}, \ldots S_{n}\right\}$ by calculating for $n$ times. The attribute value interval number $\bar{a}_{i j}=\left[a_{i j}^{L}, a_{i j}^{U}\right]$ of the $j$ th index $Q_{j}$ obtained by the $i$ th measurement composes the decision matrix of the interval numbers.

$$
\overline{\mathrm{A}}=\left[\begin{array}{cccc}
\overline{\mathrm{a}}_{11} & \overline{\mathrm{a}}_{12} & \ldots & \overline{\mathrm{a}}_{1 \mathrm{n}} \\
\overline{\mathrm{a}}_{21} & \overline{\mathrm{a}}_{22} & \ldots & \overline{\mathrm{a}}_{2 \mathrm{n}} \\
\vdots & \vdots & \ddots & \vdots \\
\overline{\mathrm{a}}_{\mathrm{m} 1} & \overline{\mathrm{a}}_{\mathrm{m} 2} & \ldots & \overline{\mathrm{a}}_{\mathrm{mn}}
\end{array}\right]
$$

Step 2: Standardize the data.

Standardize the decision matrix of interval numbers:

$$
\overline{\mathrm{P}}=\left[\begin{array}{cccc}
\overline{\mathrm{p}}_{11} & \overline{\mathrm{p}}_{12} & \ldots & \overline{\mathrm{p}}_{1 \mathrm{n}} \\
\overline{\mathrm{p}}_{21} & \overline{\mathrm{p}}_{22} & \ldots & \overline{\mathrm{p}}_{2 \mathrm{n}} \\
\vdots & \vdots & \ddots & \vdots \\
\overline{\mathrm{p}}_{\mathrm{m} 1} & \overline{\mathrm{p}}_{\mathrm{m} 2} & \cdots & \overline{\mathrm{p}}_{\mathrm{mn}}
\end{array}\right]
$$

If index $Q_{j}$ is a positive index,

$$
\overline{\mathrm{P}}_{\mathrm{ij}}=\frac{\overline{\mathrm{a}}_{\mathrm{ij}}}{\sum_{\mathrm{k}=1}^{\mathrm{m}} \overline{\mathrm{a}}_{\mathrm{kj}}}
$$

Then,

$$
\left\{\begin{aligned}
\mathrm{P}_{\mathrm{ij}}^{\mathrm{L}} & =\frac{\overline{\mathrm{a}}_{\mathrm{ij}}^{\mathrm{L}}}{\sum_{\mathrm{k}=1}^{\mathrm{m}} \mathrm{a}_{\mathrm{kj}}^{\mathrm{U}}} \\
\mathrm{P}_{\mathrm{ij}}^{\mathrm{U}} & =\frac{\overline{\mathrm{a}}_{\mathrm{ij}}^{\mathrm{U}}}{\sum_{\mathrm{k}=1}^{\mathrm{m}} \mathrm{a}_{\mathrm{kj}}^{\mathrm{L}}}
\end{aligned}\right.
$$


If index $Q_{j}$ is a negative index,

$$
\overline{\mathrm{P}}_{\mathrm{ij}}=\frac{\frac{1}{\overline{\mathrm{a}}_{\mathrm{ij}}}}{\sum_{\mathrm{k}=1}^{\mathrm{m}} \frac{1}{\overline{\mathrm{a}}_{\mathrm{kj}}}}
$$

Then,

$$
\left\{\begin{array}{l}
\mathrm{P}_{\mathrm{ij}}^{\mathrm{L}}=\frac{\frac{1}{\mathrm{a}_{\mathrm{ij}}^{\mathrm{U}}}}{\sum_{\mathrm{k}=1}^{\mathrm{m}} \frac{1}{\mathrm{a}_{\mathrm{kj}}^{\mathrm{L}}}} \\
\mathrm{P}_{\mathrm{ij}}^{\mathrm{U}}=\frac{\frac{1}{\mathrm{a}_{\mathrm{ij}}^{\mathrm{L}}}}{\sum_{\mathrm{k}=1}^{\mathrm{m}} \frac{1}{\mathrm{a}_{\mathrm{Vj}}}}
\end{array}\right.
$$

Here, $\sum_{\mathrm{k}=1}^{\mathrm{m}} \mathrm{p}_{\mathrm{ij}}^{\mathrm{L}} \leq 1 ; \sum_{\mathrm{k}=1}^{\mathrm{m}} \mathrm{p}_{\mathrm{ij}}^{\mathrm{U}} \geq 1 ; \mathrm{j}=1,2, \ldots \mathrm{n}$.

Step 3: Calculate the entropy value.

The entropy of the $j$ th index $Q_{j}$ is

$$
H_{j}=-K \sum_{i=1}^{m} P_{i j} \ln p_{i j}, j=1,2, \ldots . n .
$$

Here, $\mathrm{k}=(\operatorname{lnm})^{-1}$; assume $\mathrm{P}_{\mathrm{ij}}=0$, then $\mathrm{P}_{\mathrm{ij}} \ln \mathrm{P}_{\mathrm{ij}}=0$.

To determine the index entropy value $\overline{\mathrm{H}}_{\mathrm{j}}=\left[\mathrm{H}_{\mathrm{j}}^{\mathrm{L}}, \mathrm{H}_{\mathrm{j}}^{\mathrm{U}}\right]$, establish two optimization models:

$$
\begin{gathered}
H_{j}^{L}=\min \left\{-k \sum_{i=1}^{m} p_{i j} \ln p_{i k}\right\} \\
\text { s.t }\left\{\begin{array}{c}
p_{i j}^{L} \leq p_{i j} \leq p_{i j}^{U} \cdot i=1,2, \ldots, m \\
\sum_{i=1}^{m} p_{i j}=1
\end{array}\right.
\end{gathered}
$$

and

$$
\begin{gathered}
\mathrm{H}_{\mathrm{j}}^{\mathrm{U}}=\max \left\{-\mathrm{k} \sum_{\mathrm{i}=1}^{\mathrm{m}} \mathrm{p}_{\mathrm{ij}} \ln \mathrm{p}_{\mathrm{ik}}\right\} \\
\text { s.t }\left\{\begin{array}{c}
\mathrm{p}_{\mathrm{ij}}^{\mathrm{L}} \leq \mathrm{p}_{\mathrm{ij}} \leq \mathrm{p}_{\mathrm{ij}}^{\mathrm{U}} \cdot \mathrm{i}=1,2, \ldots, m \\
\sum_{\mathrm{i}=1}^{\mathrm{m}} \mathrm{p}_{\mathrm{ij}}=1
\end{array}\right.
\end{gathered}
$$

Step 4: Calculate the entropy weight.

In the attained interval entropy values of indices $\overline{\mathrm{H}}_{\mathrm{j}}=\left[\mathrm{H}_{\mathrm{j}}^{\mathrm{L}}, \mathrm{H}_{\mathrm{j}}^{\mathrm{U}}\right], \mathrm{j}=1,2, \ldots \mathrm{n}$, the interval entropy weight of the $j$ th index is $Q_{j}: \bar{w}_{j}=\left[w_{j}^{L}, w_{j}^{U}\right]$. Here,

$$
\bar{w}_{j}=\frac{1-\bar{H}_{j}}{n-\sum_{j=1}^{n} \bar{H}_{j}}, j=1,2, \ldots n
$$

can be written as

$$
\left\{\begin{array}{l}
w_{j}^{L}=\frac{1-H_{j}^{U}}{n-\sum_{j=1}^{n} H_{j}^{L}} \\
w_{j}^{U}=\frac{1-H_{j}^{L}}{n-\sum_{j=1}^{n} H_{j}^{U}}
\end{array}, j=1,2, \ldots n\right.
$$

Step 5: Normalize entropy weights. 
Normalize the above entropy weights:

$$
\overline{\mathrm{w}}_{\mathrm{j}}^{\prime}=\frac{\overline{\mathrm{w}}_{\mathrm{j}}}{\overline{\mathrm{w}}_{\mathrm{j}}^{+}}, \mathrm{j}=1,2, \ldots \mathrm{n}
$$

Here, $\overline{\mathrm{w}}_{\mathrm{j}}^{+}=\left[\mathrm{w}_{\mathrm{j}}^{+}, \mathrm{w}_{\mathrm{j}}^{+}\right] ; \mathrm{w}_{\mathrm{j}}^{+}=\max \left\{\mathrm{w}_{\mathrm{j}}^{\mathrm{U}} \mid j=1,2, \ldots \mathrm{n}\right\}$

can be written as

$$
\left\{\begin{array}{l}
w_{j}^{L^{\prime}}=\frac{w_{j}^{L}}{w_{j}^{+}} \\
w_{j}^{U \prime}=\frac{w_{j}^{U^{\prime}}}{w_{j}^{+}}
\end{array}, j=1,2, \ldots, n .\right.
$$

\subsection{Evaluation Method Based on Interval Cloud Model}

After weighting the indices, the cloud generator algorithm based on interval cloud data is employed to calculate the interval cloud number feature value of every index. Then the results above are applied in the evaluation. The specific process consists of steps 6 to 10 .

Step 6: Determine the comment class for assessing the interval cloud model.

Establish a set of commenting factors according to the results of the questionnaire survey by the experts ( $V$ = very weak, quite weak, general, quite strong, very strong). Quantize this set of comments to $[0,1]$ and invite industry experts to give interval values to every index (very weak, quite weak, general, quite strong, very strong). Then calculate interval expectation, entropy, and super entropy of every class of state value and role value.

Step 7: Combine interval number and cloud model. Through the uncertainty of the interval number and number features of the cloud model, we have taken ambiguous and uncertain factors in the process of measurement into consideration and achieved an effective inter-interval switch between qualitative and quantitative. The attained measurement result conforms better to our understanding of qualitative language [39]. We processed the upper and lower limits of the most possible estimated interval $\mathrm{v}^{\mathrm{k}}=\left[\mathrm{v}_{1}^{\mathrm{k}}, \mathrm{v}_{2}^{\mathrm{k}}\right], \mathrm{k}=1,2 \ldots \mathrm{m}$ given by the experts into sequences of numbers: the upper limit and lower limit sequences of numbers of the estimated interval [40].

Step 8: Establish a comment index interval matrix according to cloud model theory. Every row represents comment conclusions of a certain expert on the index, and every column represents comment conclusions of different experts on the same index.

$$
\mathrm{R}=\left[\begin{array}{cccc}
{\left[\mathrm{x}_{11}^{\mathrm{L}}, \mathrm{x}_{11}^{\mathrm{H}}\right]} & {\left[\mathrm{x}_{12}^{\mathrm{L}}, \mathrm{x}_{12}^{\mathrm{H}}\right]} & \ldots & {\left[\mathrm{x}_{1 \mathrm{n}}^{\mathrm{L}}, \mathrm{x}_{1 \mathrm{n}}^{\mathrm{H}}\right]} \\
{\left[\mathrm{x}_{21}^{\mathrm{L}}, \mathrm{x}_{21}^{\mathrm{H}}\right]} & {\left[\mathrm{x}_{22}^{\mathrm{L}}, \mathrm{x}_{22}^{\mathrm{H}}\right]} & \ldots & {\left[\mathrm{x}_{2 \mathrm{n}}^{\mathrm{L}}, \mathrm{x}_{2 \mathrm{n}}^{\mathrm{H}}\right]} \\
\vdots & \vdots & \ddots & \vdots \\
{\left[\mathrm{x}_{\mathrm{m} 1}^{\mathrm{L}}, \mathrm{x}_{\mathrm{m} 1}^{\mathrm{H}}\right]} & {\left[\mathrm{x}_{\mathrm{m} 2}^{\mathrm{L}}, \mathrm{x}_{\mathrm{m} 2}^{\mathrm{H}}\right]} & \cdots & {\left[\mathrm{x}_{\mathrm{mn}}^{\mathrm{L}}, \mathrm{x}_{\mathrm{mn}}^{\mathrm{H}}\right]}
\end{array}\right]
$$

Step 9: According to the cloud model theory, number feature values of the interval cloud model of bottom-level indices are attained:

$$
\begin{aligned}
& \mathrm{E}_{\mathrm{x}}=\left[\mathrm{E}_{\mathrm{x}}^{\mathrm{L}}, \mathrm{E}_{\mathrm{x}}^{\mathrm{H}}\right]=\frac{1}{\mathrm{~m}} \sum_{\mathrm{i}=1}^{\mathrm{m}}\left[\mathrm{x}_{\mathrm{i}}^{\mathrm{L}}, \mathrm{x}_{\mathrm{i}}^{\mathrm{H}}\right] \\
& E_{n}=\left[E_{n}^{L}, E_{n}^{H}\right]=\sqrt{\frac{\pi}{2}} \times \frac{1}{m} \sum_{i=1}^{m}\left|\left[x_{i}^{L}, x_{i}^{H}\right]-\left[E_{i}^{L}, E_{i}^{H}\right]\right| \\
& \mathrm{H}_{\mathrm{e}}=\left[\mathrm{H}_{\mathrm{e}}^{\mathrm{L}}, \mathrm{H}_{\mathrm{e}}^{\mathrm{H}}\right]=\sqrt{\frac{1}{\mathrm{~m}-1} \sum_{\mathrm{i}=1}^{\mathrm{m}}\left(\left[\mathrm{x}_{\mathrm{i}}^{\mathrm{L}}, \mathrm{x}_{\mathrm{i}}^{\mathrm{H}}\right]-\left[\mathrm{E}_{\mathrm{i}}^{\mathrm{L}}, \mathrm{E}_{\mathrm{i}}^{\mathrm{H}}\right]\right)^{2}-\mathrm{E}_{\mathrm{n}}^{2}}
\end{aligned}
$$


Step 10: Use all of the attained interval weight values of bottom-level indices to get interval comment results of upper-level indices according to the rules of interval number calculation. Hence, the interval cloud number feature value of every index is attained:

$$
\left\{\begin{array}{c}
E_{x}=\frac{E_{x_{1}} w_{1}+E_{x_{2}} w_{2}+\ldots+E_{x_{n}} w_{n}}{w_{1}+w_{2}+\ldots+w_{n}} \\
E_{n}=\frac{w_{1}^{2}}{w_{1}^{2}+w_{2}^{2}+\ldots+w_{n}^{2}} E_{n 1}+\ldots+\frac{w_{n}^{2}}{w_{1}^{2}+w_{2}^{2}+\ldots+w_{n}^{2}} E_{n n} \\
H_{e}=\frac{w_{1}^{2}}{w_{1}^{2}+w_{2}^{2}+\ldots+w_{n}^{2}} H_{e 1}+\ldots+\frac{w_{n}^{2}}{w_{1}^{2}+w_{2}^{2}+\ldots+w_{n}^{2}} H_{e n}
\end{array}\right.
$$

\subsection{NMSI Model}

The NMSI (niche-mutualism-shift-integration) model is constructed based on grounded theory [41]. This model describes the path of the evolution of the innovation ecosystem from the simplex model to the complex model. It mainly includes four stages: niche, mutualism, shift, and integration. At the same time, factors, such as external support, adaptive behavior, organizational change, and dynamic capabilities, play decisive roles in these four phases. The whole process is shown in Figure 4.

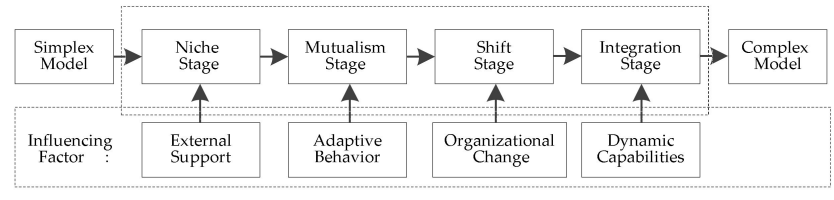

Figure 4. NMSI model for evolution of the innovation ecosystem.

\section{Results}

\subsection{Index Weight}

We invited 10 industry experts to score the importance of every index in the index system, and the score range is $[0,10]$. To better assess the importance, every expert presented the importance of a certain index with interval numbers. We sorted out the interval scores given by the experts and constructed an interval judgment matrix of the index system. Moreover, we determined the interval weight of every index according to the steps stated above, shown in Table 1.

Table 1. Interval weight of every index of photovoltaic agriculture niche evaluation system.

\begin{tabular}{|c|c|c|c|c|}
\hline Target Layer & Criterion Layer & Weight & Index Layer & Weight \\
\hline \multirow{12}{*}{ Niche state (S) } & \multirow{4}{*}{$\begin{array}{l}\text { Niche breadth } \\
\qquad\left(\mathrm{S}_{1}\right)\end{array}$} & \multirow{4}{*}{$0.2016-0.2337$} & Coverage rate $\left(\mathrm{S}_{11}\right)$ & $0.0531-0.0822$ \\
\hline & & & Cooperation rate $\left(S_{12}\right)$ & $0.1214-0.1226$ \\
\hline & & & Environmental adaptation degree $\left(\mathrm{S}_{13}\right)$ & $0.0084-0.0108$ \\
\hline & & & Utilization of resources $\left(\mathrm{S}_{14}\right)$ & $0.0163-0.0205$ \\
\hline & \multirow{4}{*}{$\begin{array}{c}\text { Niche overlap } \\
\left(\mathrm{S}_{2}\right)\end{array}$} & \multirow{4}{*}{$0.2420-0.2533$} & Sunlight utilization ratio $\left(S_{21}\right)$ & $0.1412-0.1418$ \\
\hline & & & Land utilization rate $\left(\mathrm{S}_{22}\right)$ & $0.0688-0.0803$ \\
\hline & & & Resource input $\left(\mathrm{S}_{23}\right)$ & $0.0165-0.0172$ \\
\hline & & & Human input $\left(\mathrm{S}_{24}\right)$ & $0.0142-0.0154$ \\
\hline & \multirow{4}{*}{$\begin{array}{l}\text { Niche advantage } \\
\left(\mathrm{S}_{3}\right)\end{array}$} & \multirow{4}{*}{$0.1609-0.1695$} & Technical advantages $\left(\mathrm{S}_{31}\right)$ & $0.0718-0.0757$ \\
\hline & & & Policy advantages $\left(\mathrm{S}_{32}\right)$ & $0.0682-0.0758$ \\
\hline & & & Cost advantages $\left(\mathrm{S}_{33}\right)$ & $0.0102-0.0105$ \\
\hline & & & Sunshine resource advantage $\left(\mathrm{S}_{34}\right)$ & $0.0075-0.0107$ \\
\hline \multirow{12}{*}{ Niche role (R) } & \multirow{4}{*}{$\begin{array}{l}\text { Economic niche } \\
\qquad\left(\mathrm{R}_{1}\right)\end{array}$} & \multirow{4}{*}{$0.1413-0.1486$} & GDP growth rate $\left(\mathrm{R}_{11}\right)$ & $0.0174-0.0227$ \\
\hline & & & Power generation $\left(\mathrm{R}_{12}\right)$ & $0.0281-0.0400$ \\
\hline & & & Crop economic benefit $\left(R_{13}\right)$ & $0.0762-0.0807$ \\
\hline & & & Tourism economy $\left(\mathrm{R}_{14}\right)$ & $0.0099-0.0150$ \\
\hline & \multirow{4}{*}{$\begin{array}{l}\text { Environmental } \\
\text { niche }\left(R_{2}\right)\end{array}$} & \multirow{4}{*}{$0.1068-0.1184$} & $\mathrm{CO}_{2}$ emission reduction $\left(\mathrm{R}_{21}\right)$ & $0.0377-0.0504$ \\
\hline & & & $\mathrm{SO}_{2}$ emission reduction $\left(\mathrm{R}_{22}\right)$ & $0.0203-0.0283$ \\
\hline & & & Saving standard coal $\left(\mathrm{R}_{23}\right)$ & $0.0099-0.0101$ \\
\hline & & & Blocking wave adjusting temperature $\left(R_{24}\right)$ & $0.0297-0.0387$ \\
\hline & \multirow{4}{*}{ Social niche $\left(R_{3}\right)$} & \multirow{4}{*}{$0.1081-0.1158$} & Alleviating contradiction of electric power $\left(\mathrm{R}_{31}\right)$ & $0.0078-0.0097$ \\
\hline & & & Optimizing agricultural and energy structure $\left(\mathrm{R}_{32}\right)$ & $0.0381-0.0465$ \\
\hline & & & Providing employment $\left(\mathrm{R}_{33}\right)$ & $0.0156-0.0196$ \\
\hline & & & Promoting tourism development $\left(\mathrm{R}_{34}\right)$ & $0.0465-0.0490$ \\
\hline
\end{tabular}




\subsection{Evaluation Results}

Expert scoring was applied to determine values. We took the interval scoring values given by the 10 experts on the quantitative index interval values that went through standardization into Equations (14)-(17) and combined them with the comment index interval matrix of the index level to attain interval expected values, entropy, and super entropy of every index in the index level, as shown in Table 2.

Table 2. Number feature value of every index in the interval cloud model.

\begin{tabular}{|c|c|c|c|c|c|}
\hline Target Layer & $\begin{array}{c}\text { Criterion } \\
\text { Layer }\end{array}$ & Index & $\begin{array}{l}\text { Expected } \\
\text { Value }\end{array}$ & Entropy & $\begin{array}{l}\text { Super } \\
\text { Entropy }\end{array}$ \\
\hline \multirow{12}{*}{$\begin{array}{l}\text { Niche state } \\
\text { (S) }\end{array}$} & \multirow{4}{*}{$\begin{array}{l}\text { Niche breadth } \\
\quad\left(\mathrm{S}_{1}\right)\end{array}$} & Coverage rate $\left(S_{11}\right)$ & $0.4570-0.5500$ & $0.0356-0.0376$ & $0.0055-0.0056$ \\
\hline & & Cooperation rate $\left(\mathrm{S}_{12}\right)$ & $0.6840-0.7460$ & $0.0847-0.0902$ & $0.0125-0.0127$ \\
\hline & & Environmental adaptation degree $\left(\mathrm{S}_{13}\right)$ & $0.4630-0.5340$ & $0.0892-0.0990$ & $0.0121-0.0142$ \\
\hline & & Utilization of resources $\left(\mathrm{S}_{14}\right)$ & $0.5920-0.6570$ & $0.0607-0.0677$ & $0.0081-0.0102$ \\
\hline & \multirow{4}{*}{$\begin{array}{l}\text { Niche overlap } \\
\quad\left(\mathrm{S}_{2}\right)\end{array}$} & Sunlight utilization ratio $\left(\mathrm{S}_{2}\right.$ & $0.6870-0.7560$ & $0.0782-0.0882$ & $0.0123-0.0133$ \\
\hline & & Land utilization rate $\left(\mathrm{S}_{22}\right)$ & $0.7110-0.7930$ & $0.1141-0.1258$ & $0.0153-0.0166$ \\
\hline & & Resource input $\left(\mathrm{S}_{23}\right)$ & $0.6510-0.7270$ & $0.0614-0.0692$ & $0.0098-0.0112$ \\
\hline & & Human input $\left(\mathrm{S}_{24}\right)$ & $0.4780-0.5600$ & $0.0777-0.1053$ & $0.0099-0.0139$ \\
\hline & \multirow{4}{*}{$\begin{array}{c}\text { Niche } \\
\text { advantage }\left(\mathrm{S}_{3}\right)\end{array}$} & Technical advantages $\left(\mathrm{S}_{31}\right)$ & $0.4530-0.5180$ & $0.0852-0.0922$ & $0.0111-0.0126$ \\
\hline & & Policy advantages $\left(\mathrm{S}_{32}\right)$ & $0.6000-0.6950$ & $0.0551-0.0639$ & $0.0090-0.0105$ \\
\hline & & Cost advantages $\left(\mathrm{S}_{33}\right)$ & $0.6580-0.7310$ & $0.0815-0.0902$ & $0.0110-0.0127$ \\
\hline & & Sunshine resource advantage $\left(\mathrm{S}_{34}\right)$ & $0.5900-0.6730$ & $0.0664-0.0702$ & $0.0101-0.0106$ \\
\hline \multirow{12}{*}{ Niche role (R) } & \multirow{4}{*}{$\begin{array}{l}\text { Economic niche } \\
\qquad\left(\mathrm{R}_{1}\right)\end{array}$} & GDP growth rate $\left(R_{11}\right)$ & $0.6210-0.6930$ & $0.0449-0.0566$ & $0.0066-0.0075$ \\
\hline & & Power Generation & $0.6570-0.7470$ & $0.0614-0.0622$ & $0.0079-0.0087$ \\
\hline & & Crop economic benefit $\left(\mathrm{R}_{13}\right)$ & $0.6580-0.7430$ & $0.0574-0.0707$ & $0.0090-0.0103$ \\
\hline & & Tourism economy $\left(\mathrm{R}_{14}\right)$ & $0.4040-0.4890$ & $0.0892-0.0963$ & $0.0123-0.0135$ \\
\hline & \multirow{4}{*}{$\begin{array}{l}\text { Environmental } \\
\text { niche }\left(\mathrm{R}_{2}\right)\end{array}$} & $\mathrm{CO}_{2}$ emission reduction $\left(\mathrm{R}_{21}\right)$ & $0.6430-0.7200$ & $0.0802-0.0840$ & $0.0111-0.0118$ \\
\hline & & $\mathrm{SO}_{2}$ emission reduction $\left(\mathrm{R}_{22}\right)$ & $0.5430-0.6200$ & $0.0802-0.0840$ & $0.0111-0.0118$ \\
\hline & & Saving standard coal $\left(\mathrm{R}_{23}\right)$ & $0.6150-0.6950$ & $0.1065-0.1103$ & $0.0142-0.0144$ \\
\hline & & Blocking wave adjusting temperature $\left(\mathrm{R}_{24}\right)$ & $0.4680-0.5630$ & $0.0915-0.0978$ & $0.0129-0.0138$ \\
\hline & \multirow{4}{*}{$\begin{array}{l}\text { Social niche } \\
\quad\left(\mathrm{R}_{3}\right)\end{array}$} & $\begin{array}{l}\text { Alleviating contradiction of electric } \\
\text { power }\left(R_{31}\right)\end{array}$ & $0.5350-0.6150$ & $0.0940-0.1028$ & $0.0124-0.0137$ \\
\hline & & $\begin{array}{l}\text { Optimizing agricultural and energy } \\
\text { structure }\left(R_{32}\right)\end{array}$ & $0.6940-0.7760$ & $0.0436-0.0491$ & $0.0067-0.0070$ \\
\hline & & Providing employment $\left(\mathrm{R}_{33}\right)$ & $0.4800-0.5780$ & $0.0551-0.0602$ & $0.0085-0.0085$ \\
\hline & & Promoting tourism development $\left(\mathrm{R}_{34}\right)$ & $0.3520-0.4060$ & $0.0812-0.0897$ & $0.0103-0.0117$ \\
\hline
\end{tabular}

We calculated the interval cloud model number features of the indices according to Equation (18), as shown in Table 3. We used Matlab 9.1 to present interval feature values of indices in Table 3 with the interval cloud picture, as shown in Figures 5 and 6.

Table 3. Interval cloud model number features of indices.

\begin{tabular}{cccc}
\hline Index & Expected Value & Entropy & Super Entropy \\
\hline Niche breadth $\left(\mathrm{S}_{1}\right)$ & $0.6049-0.6616$ & $0.0662-0.0745$ & $0.0098-0.0105$ \\
Niche overlap $\left(\mathrm{S}_{2}\right)$ & $0.6796-0.7529$ & $0.0905-0.0962$ & $0.0132-0.0138$ \\
Niche advantage $\left(\mathrm{S}_{3}\right)$ & $0.5375-0.6168$ & $0.0745-0.0747$ & $0.0107-0.0109$ \\
Economic niche $\left(\mathrm{R}_{1}\right)$ & $0.6340-0.7126$ & $0.0604-0.0684$ & $0.0090-0.0096$ \\
Environmental niche $\left(\mathrm{R}_{2}\right)$ & $0.5727-0.6418$ & $0.0868-0.0896$ & $0.0121-0.0125$ \\
Social niche $\left(\mathrm{R}_{3}\right)$ & $0.5164-0.5766$ & $0.0658-0.0714$ & $0.0089-0.0097$ \\
Niche state $(\mathrm{S})$ & $0.6169-0.6850$ & $0.0776-0.0833$ & $0.0113-0.0120$ \\
Niche role $(\mathrm{R})$ & $0.5784-0.6513$ & $0.0698-0.0760$ & $0.0099-0.0106$ \\
Integrated niche & $0.6025-0.6728$ & $0.0748-0.0806$ & $0.0108-0.0115$ \\
\hline
\end{tabular}



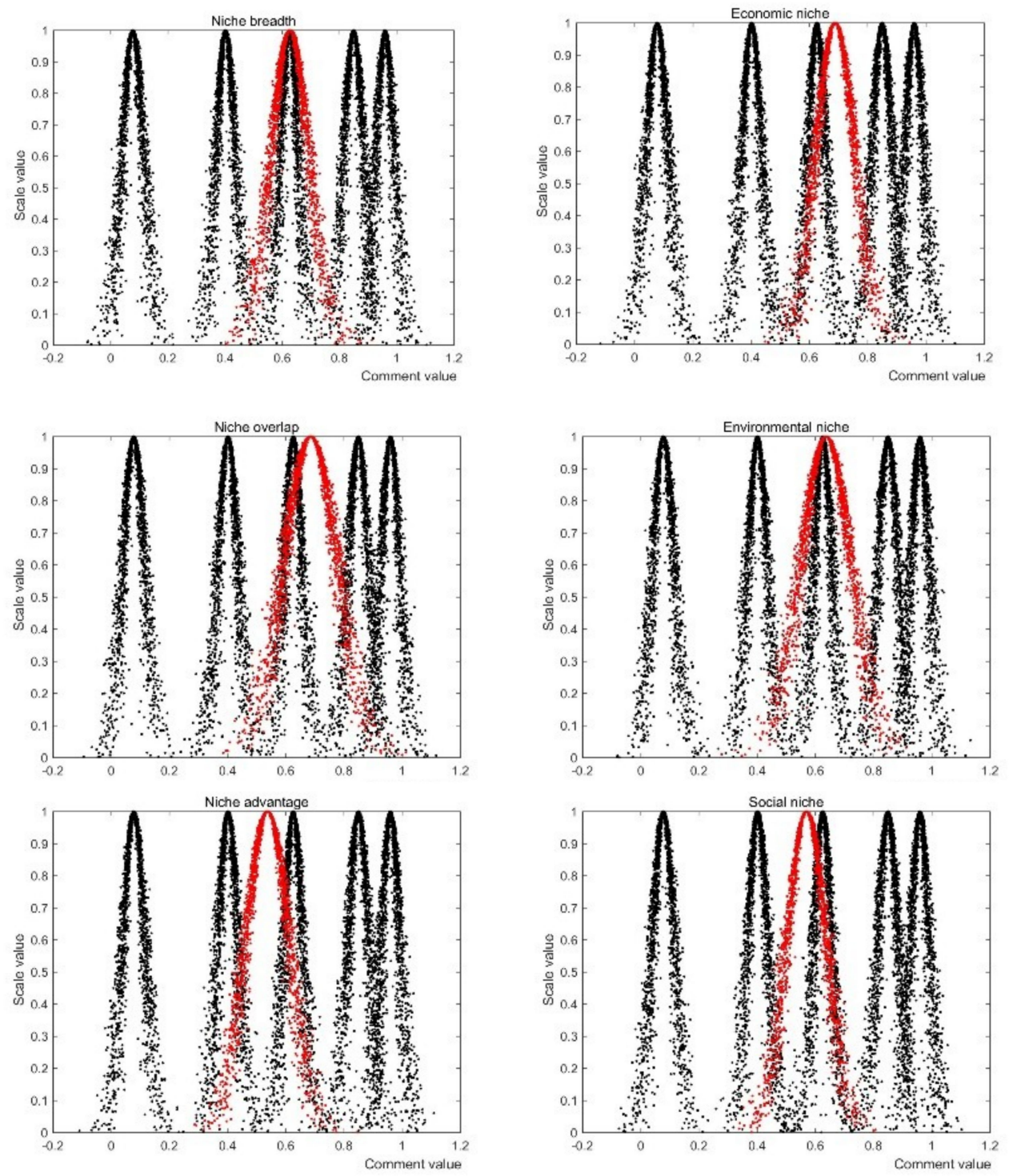

Figure 5. Comment grade interval cloud model of every index in criterion layer. 

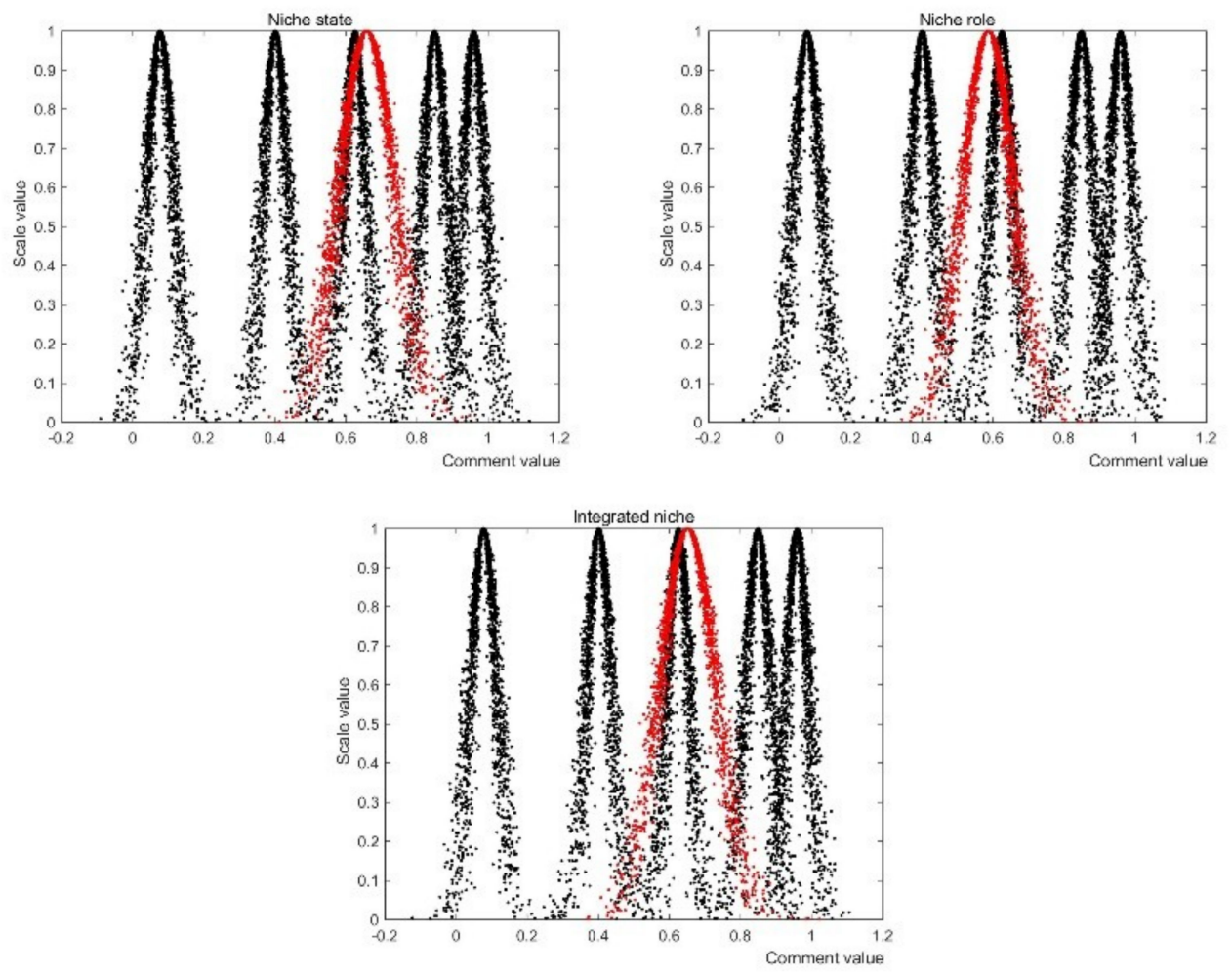

Figure 6. Interval cloud model of target comment.

\subsection{Development Stage of Ecological Niche of China's Photovoltaic Agriculture}

The results of evaluating the ecological niche of photovoltaic agriculture showed that the niche state and role values of this system remain in the medium range, which means photovoltaic agriculture projects are developing well in China at present, and there are bright prospects and huge potential for expansion in the future. Despite that, integrating the two industries cannot be accomplished in one action. On the contrary, the best state can only be achieved through a series of long periods. The authors of this paper, by applying grounded theory and method referring to the NMSI model constructed by Guo [41] and combining it with features of the niche state and niche role of photovoltaic agriculture, conclude that photovoltaic agriculture in China will go through four stages in the process of development in order to achieve transformation from a simplex model to a complex model. In general, the four stages are positioning, integration, leap, and symbiosis of the ecological niche. As shown in Figure 7, the curve shows a trend of going flat before rising sharply. This is because the impact of the early stage in the current state is more obvious in the development process, which is shown as a lower slope in the early stage. When the development of the system enters the middle and later periods, there will be a great potential that will affect the development in the future, which is shown as a bigger slope in the chart in the middle and later periods. Incorporating the measurement results of niche state and niche role values in Section 4.2 into the development trend chart, it can be intuitively found that the integrated niche of China's photovoltaic agriculture is currently between the positioning stage and the integration stage, as shown by the star in Figure 7. 


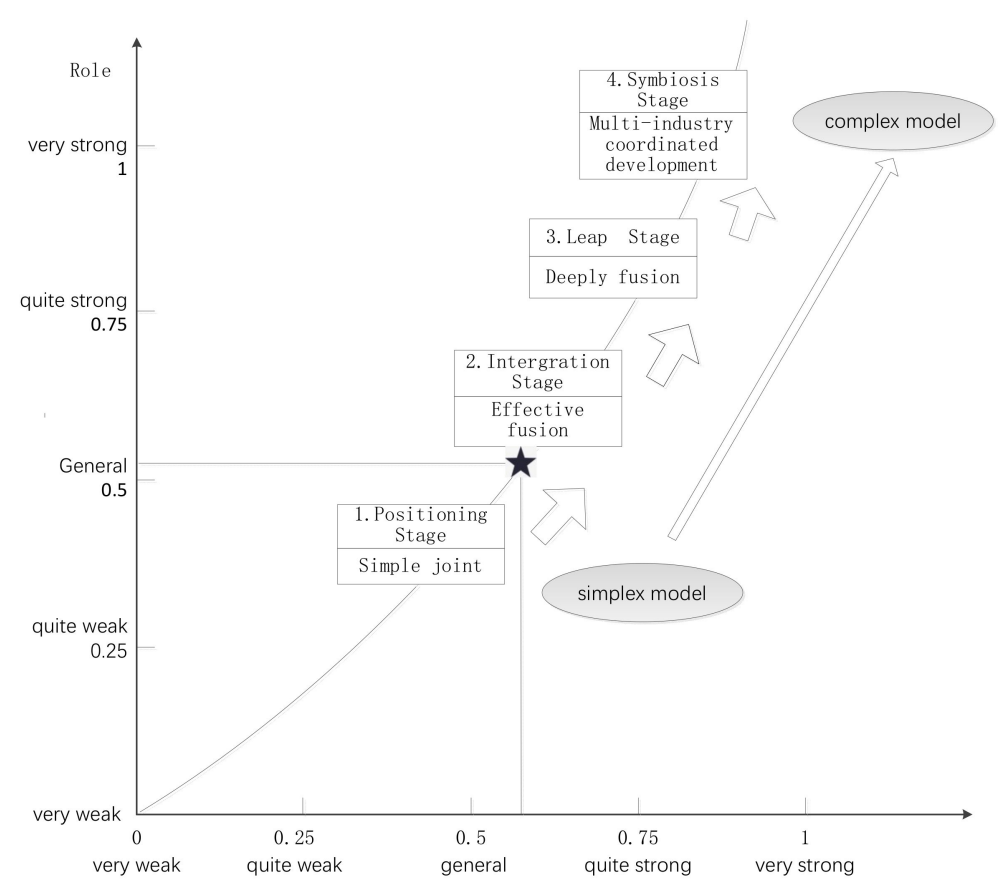

Figure 7. Development trend chart of integrated niche of China's photovoltaic agriculture.

\section{Discussion}

\subsection{Niche State}

As shown by feature values of the state aspect of the ecological niche and cloud pictures, China's photovoltaic agriculture is developing well so far. As far as specific influencing factors are concerned, the expected values of niche breadth and overlap are in a generally good state. However, the value of a niche advantage lies between "quite weak" and "general." There are several reasons for that. First, regarding the cooperation rate $(0.6840-0.7460,0.0847-0.0902,0.0125-0.0127)$, photovoltaic agriculture is composed of agriculture and photovoltaics in an organic way. The precondition for the smooth development of this system is whether the two completely different industries can join and cooperate effectively. So far, the two industries in China's photovoltaic agriculture can cooperate well. In general, they have achieved a state where electricity is generated above, and planting and breeding are conducted below. That is, electricity generation co-exists with planting and breeding. Hence, there is a situation where resources are shared, dimensional, and recycling development is achieved, and multiple parties are winning, and a high degree of cooperation is developed. Second, in terms of technological advantages (0.4530-0.5180, 0.0852-0.0922, 0.0111-0.0126), effective technology is the foundation for achieving complementarity between agriculture and photovoltaics. In foreign countries, photovoltaic agriculture has become quite mature, with all technologies reaching a high level. In China, however, the project is in the early stage [42], and relevant technologies are not mature [4], which affects the ecological niche of the whole system. Paying attention to updating and developing technology and making maximal use of sunlight and land resources through a reasonable design has become indispensable for the healthy operation of the system. Third, policy advantages (0.6000-0.6950, 0.0551-0.0639, 0.0090-0.0105). Photovoltaic agriculture requires capital, land, and other resources. Hence, great support from the government is indispensable. To sum up, policy inclination has a great impact on the ecological niche of the system. As far as China's current situation is concerned, governments at all levels are attaching great importance to the comprehensive utilization of photovoltaic resources, proactively issuing supportive policies and vigorously developing the photovoltaic and agricultural industries [43,44]. Consequently, great achievements have been made. So far, photovoltaic agriculture is still in the early construction period in China, and support from 
relevant government policies is moderate but not standardized. If standardization continues, support from government policies will increase, the two industries will be combined with clean energy policies of the country, efforts will be made to transform the form of economic development, the industrial structure will be constantly optimized, and the speed of transforming traditional agriculture to modern agriculture will increase, the development of photovoltaic agriculture projects will be facilitated, and favorable social, economic, and environmental benefits will be generated.

\subsection{Niche Role}

As shown by the roles of feature values of the ecological niche and cloud pictures, the niche role value of China's photovoltaic agriculture is in a good but weak state. Based on an analysis of specific influencing factors, the expected values of economic and environmental niche are generally good, but the expected value of social niche is in a generally weak state. There are several reasons for that. First, $\mathrm{CO}_{2}$ emission reduction $(0.6430-0.7200,0.0802-0.0840,0.0111-0.0118)$. The environmental pollution of solar power generation is zero. Meanwhile, it can offset greenhouse gas emissions caused by the consumption of traditional energy. Hence, solar power is the future direction for the development of energy. Photovoltaics, as a clean energy industry, can minimize its degree of pollution on the environment. Similarly, photovoltaic agriculture also has the advantage of clean energy. Reduced carbon dioxide emission has played an important role in the niche and has resulted in a very good effect [3]. Second, it promotes optimization of the agricultural and energy structure $(0.6940-0.7760$, $0.0436-0.0491,0.0067-0.0070$ ). From the perspective of weight, photovoltaic agriculture projects can facilitate adjustments to the energy structure as well as upgrade and optimize the structure of the agricultural industry. From actual measurement results, China's photovoltaic agriculture projects have actually facilitated adjustments to the energy and agriculture structure, though the degree of effort is not obvious. In other words, there is still potential for development in this area. Hence, it is necessary to achieve the state of double income through innovative, intelligent photovoltaics plus scientific and technological agriculture to respond to changes to industrial policies. Third, promoting the development of tourism $(0.3520-0.4060,0.0812-0.0897,0.0103-0.0117)$. As far as index weight is concerned, photovoltaic agriculture plays an important facilitating role in the development of the tourism industry, though the actual value is relatively small, which means China's photovoltaic agriculture projects are in the preliminary stage and only a simple combination of agriculture and photovoltaics is achieved. Meanwhile, the system has not expanded to other industries like tourism [42]. Therefore, it may lean toward tourism in future development to achieve a multiple-win where "one land is for three usages and light for three usages," that is, power generation, planting, and tourism.

\subsection{Integrated Niche}

Ecological niche positioning is the stage where agriculture is simply connected to photovoltaics. In the early stage of photovoltaic agriculture formation, the system is shown as having a strong awareness of self-protection, ambiguous willingness to integrate, and a lack of an integrating the technology paradigm. Due to a lack of experience [3], agriculture and photovoltaics are placed on the same land, and the relevance between the two industries is not fully understood. Hence, the system enters a stage where the two industries are connected in a simple way. The integration stage is where agriculture and photovoltaics are integrated effectively. As the relationship between the industries gets deeper, the two ecological niches-agriculture and photovoltaics-will generate competition and cooperation through the integration of knowledge, information, and resources. Through constant cooperation over time, the two industries will get to know and accept each other. Through the effective integration of resources, they will enter the stage of effective integration. Finally, a win-win state of generating power above and planting and breeding below will be achieved. The leap stage is where the two industries have deep integration. As the ecological niche constantly differentiates and overlaps, agriculture and photovoltaics will achieve a leap by changing the niche breadth. In this stage, the combination of the two industries deepen over time and they can provide resources for each other 
upon the foundation that external resources are shared. Consequently, synergistic development is formed, and a synergistic effect is created. When the synergistic effect reaches a certain intensity, the system will automatically form a more ordered, effective, and competitive self-organized state. The symbiosis stage is where multiple industries develop synergistically. The nature of symbiosis theory is that all symbiotic units coordinate and cooperate to achieve a win-win state, which is also the goal of photovoltaic agriculture. With the leap of the ecological niche, the two industries reach a state of deep integration, so people can consider introducing other industries into photovoltaic agriculture to form an industrial symbiont and promote the optimization and upgrading of its structure. In this stage the first, second, and third industries can be connected, giving rise to a new type of business operation, new value can be created, and finally, an ordered system featuring an innovative ecological chain structure and sustainable development can be formed [4].

\section{Conclusions and Suggestions}

Based on the niche theory, we have pointed out that China's photovoltaic agriculture is based on a real ecological niche. The two industries have constantly reconstructed their resources, technology, and environmental rules in the process of integration, broken through the niche breadth of original industries, absorbed more potential resources, and achieved the formation of an ecological niche system. By combining the interval entropy weight method and interval cloud model based on the form of the interval data table, this paper comments on the ecological niche of photovoltaic agriculture.

Conclusions can be drawn as follows:

(1) Factors that affect the ecological niche of photovoltaic agriculture are the niche breadth, overlap, and advantage, and the economic, environmental, and social niches. The first three belong to the state aspect of the ecological niche of photovoltaic agriculture, while the last three belong to the role aspect of photovoltaic agriculture.

(2) Employing the method based on the interval cloud model, the niche state and role values of photovoltaic agriculture are calculated. It shows that Chinese photovoltaic agriculture is developing well so far from the perspective of niche state, and is in a good but weak state from the perspective of niche role.

(3) Based on niche state and role values, this paper reveals the four-stage evolution process of China's photovoltaic agriculture: the positioning, integration, leap, and symbiosis of ecological niche. Now China has completed the stage of positioning and entered the stage of integrating the ecological niche.

When the measurement results of state value and role value are included in the development trend chart of the niche of photovoltaic agriculture, it can be discovered intuitively that China has completed the stage of positioning and entered the stage of integration. When the two isolated industries have achieved connection to a certain degree, they will form effective integration through complete integration of knowledge, information, and resources. In this case, a win-win scenario of generating power above and planting and breeding below will be achieved. The question is how to make photovoltaic agriculture go through the second development stage smoothly and successfully step into the third stage. According to the measurement of various factors that affect niche mentioned above, the authors have come up with the following suggestions:

(1) Closely combine the most advanced photovoltaic power generation technology with agricultural technology while focusing on high efficiency to constantly improve the level of industrial integration and form a new photovoltaic agriculture and recycling ecosystem. Meanwhile, cooperate with multiple parties, such as agricultural investment companies, scientific research institutions, and agricultural schools, to provide guarantees for photovoltaic agriculture in agricultural infrastructure, agricultural technology, and photovoltaic technology.

(2) Constantly improve the level of cooperation between the agriculture and photovoltaic industries and closely relate modern agriculture with photovoltaics to make the means of industrial production and the industrialized development of agriculture integrate with each other. Furthermore, stick to the new goal and direction of current social development, combined with the development characteristics of 
the new economy, such as green and environmental development, recycling economy, and sustainable development, to gradually achieve innovation in technology and management, upgrade traditional industries, and vigorously promote the development of emerging industries.

(3) Take economic crops with ornamental value and photovoltaic power generation devices as characteristic landscape elements and construct projects for tourists to visit and study. In this way, the tourism industry will be developed, synergistic development among several industries, such as photovoltaics, agriculture, and tourism, can be achieved, and a new industrial chain and economic growth point will be formed. In addition, three-dimensional added value and utilization of land, as well as a perfect combination of high-end agriculture and new energy, can be achieved and a green and sustainable path will be developed gradually.

By considering photovoltaic agriculture as the combination of agriculture and photovoltaic industries as an object of research, this paper comments on the ecological niche of this system. The research conclusion has significant meaning for the healthy and ordered development of China's photovoltaic agriculture and plays an important role in driving the system to bring about better economic, environmental, and social effects and to achieve sustainable development of the economy and the environment.

Author Contributions: Conceptualization, L.W. and Y.W.; methodology, L.W.; validation, J.C.; formal analysis, L.W.; investigation, J.C.; data curation, J.C.; writing—original draft preparation, L.W.; writing-review and editing, J.C.'

Funding: This research was funded by the National Natural Science Foundation of China in 2018 (grant no. 71873064), General Projects of Humanities and Social Sciences of the Ministry of Education (Planning Projects) in 2018 (grant nos. 18YJA790085), Philosophy and Social Science Research Foundation of the Higher Education Institution of Jiangsu Province (grant nos. 2018SJA0135, 2018SJA2107), Youth Science and Technology Innovation Fund of Nanjing Forestry University (grant no. CX2017016).

Conflicts of Interest: The authors declare no conflict of interest regarding the publication of this paper.

\section{References}

1. Dinesh, H.; Pearce, J.M. The potential of agrivoltaic systems. Renew. Sustain. Energy Rev. 2016, 54, 299-308. [CrossRef]

2. Brudermann, T.; Reinsberger, K.; Orthofer, A.; Kislinger, M.; Posch, A. Photovoltaics in agriculture: A case study on decision making of farmers. Energy Policy 2013, 61, 96-103. [CrossRef]

3. Li, C.S.; Wang, H.Y.; Miao, H.; Ye, B. The economic and social performance of integrated photovoltaic and agricultural greenhouses systems: Case study in China. Appl. Energy 2017, 190, 204-212. [CrossRef]

4. Wang, T.Y.; Wu, G.X.; Chen, J.W.; Cui, P.; Chen, Z.; Yan, Y.; Zhang, Y.; Li, M.; Niu, D.; Li, B.; et al. Integration of solar technology to modern greenhouse in China: Current status, challenges and prospect. Reneww. Sustain. Energy Rev. 2017, 70, 1178-1188. [CrossRef]

5. Hutchinson, G.E. A Treatise on Limnology. Geography, Physics, and Chemistry; John Wiley \& Sons: New York, NY, USA, 1957; Volume 1, ISBN 978-047-142-569-4.

6. Hommels, A.; Peters, P.; Bijker, W.E. Techno therapy or nurtured niches? Technology studies and the evaluation of radical innovations. Res. Policy 2007, 36, 1088-1099. [CrossRef]

7. Smith, A.; Voß, J.P.; Grin, J. Innovation studies and sustainability transitions: The allure of the multi-level perspective and its challenges. Res. Policy 2010, 39, 435-448. [CrossRef]

8. Seol, H.; Park, G.; Lee, H.; Yoon, B. Demand forecasting for new media services with consideration of competitive relationships using the competitive Bass model and the theory of the niche. Technol. Forecast. Soc. Chang. 2012, 79, 1217-1228. [CrossRef]

9. Singh, J.V. Organizational Niches and the Dynamics of Organizational Founding. Organ. Sci. 1994, 5, 483-501. [CrossRef]

10. Hannan, M.T.; Carroll, G.R.; Pólos, L. The Organizational Niche. Sociol. Theory 2003, 21, 309-340. [CrossRef]

11. Hamarat, B.; Kasimoglu, M. Niche overlap-Competition and homogeneity in the organizational clusters of hotel population. Manag. Res. News 2003, 26, 60-77. [CrossRef]

12. Zhu, C.Q. The Niche Ecostate-Ecorole Theory and Expansion Hypothesis. Acta Ecol. Sin. 1997, 17, 324-332. [CrossRef] 
13. Zhang, G.Y.; Xie, W.H.; Liu, Y.; Deng, X.F. Theory and Practice of Strategic Niche Management; Science Press: Beijing, China, 2015; ISBN 978-703-042-644-4.

14. Song, Y.F.; You, J.X.; Shao, L.N.; Guo, B. Efficiency Evaluation of Niche Status of Electric Vehicle Enterprises. J. Tongii Univ. (Nat. Sci.) 2015, 43, 951-957. [CrossRef]

15. Shi, B.; Tian, H.N. Research on the Path Selection of Green Process Innovation of Home Appliances Manufacturing Based on Niche Ecostate-ecorole Theory. Manag. Rev. 2018, 2, 83-93. [CrossRef]

16. Mokyr, J. The Lever of Riches: Technological Creativity and Economic Progress; Oxford University Press: London, UK, 1990; ISBN 978-019-506-113-0.

17. Van den Belt, H.; Rip, A. The Nelson-Winter-Dosi Model and Synthetic Dye Chemistry; MIT Press: Cambridge, MA, USA, 1987; ISBN 978-026-202-262-0.

18. Smith, A.; Raven, R. What is protective space? Reconsidering niches in transitions to sustainability. Res. Policy 2012, 41, 1025-1036. [CrossRef]

19. Pigford, A.-A.E.; Hickey, G.M.; Klerkx, L. Beyond agricultural innovation systems? Exploring an agricultural innovation ecosystems approach for niche design and development in sustainability transitions. Agric. Syst. 2018, 164, 116-121. [CrossRef]

20. Marrou, H.; Wery, J.; Dufour, L.; Dupraz, C. Productivity and radiation use efficiency of lettuces grown in the partial shade of photovoltaic panels. Eur. J. Agron. 2013, 44, 54-66. [CrossRef]

21. Allardyce, C.S.; Fankhauser, C.; Zakeeruddin, S.M.; Grätzel, M.; Dyson, P.J. The influence of greenhouse-integrated photovoltaics on crop production. Sol. Energy 2017, 155, 517-522. [CrossRef]

22. Liu, W.; Liu, L.; Guan, C.; Zhang, F.; Li, M.; Lv, H.; Yao, P.; Ingenhoff, J. A novel agricultural photovoltaic system based on solar spectrum separation. Sol. Energy 2018, 162, 84-94. [CrossRef]

23. Malu, P.R.; Sharma, U.S.; Pearce, J.M. Agrivoltaic potential on grape farms in India. Sustain. Energy Technol. Assess. 2017, 23, 104-110. [CrossRef]

24. Mehmood, A.; Waqas, A.; Mahmood, H.T. Economic Viability of Solar Photovoltaic Water Pump for Sustainable Agriculture Growth in Pakistan. Mater. Today Proc. 2015, 2, 5190-5195. [CrossRef]

25. Sacchelli, S.; Garegnani, G.; Geri, F.; Grilli, G.; Paletto, A.; Zambelli, P.; Ciolli, M.; Vettorato, D. Trade-off between photovoltaic systems installation and agricultural practices on arable lands: An environmental and socio-economic impact analysis for Italy. Land Use Policy 2016, 56, 90-99. [CrossRef]

26. Jones, M.A.; Odeh, I.; Haddad, M.; Mohammad, A.H.; Quinn, J.C. Economic analysis of photovoltaic (PV) powered water pumping and desalination without energy storage for agriculture. Desalination 2016, 387, 35-45. [CrossRef]

27. Zheng, S.Z.; Wu, Q.H.; Wang, H.B. General Ecology: Principles, Methods, and Applications; Fudan University Press: Shanghai, China, 1994; ISBN 7-309-01275-5/Q.43.

28. Hannan, M.T.; Freeman, J. Organizational Ecology; Harvard University Press: Cambridge, UK, 1993; ISBN 978-067-464-349-9.

29. Hurlbert, S.H. The Measurement of Niche Overlap and Some Relatives. Ecology 1978, 59, 67-77. [CrossRef]

30. Hu, Q.Z.; Zhang, W.H. Research on Interval Number Theory and Its Application; Higher Education Press: Beijing, China, 2010; ISBN 978-703-028-993-3.

31. Song, P.; Liang, J.Y.; Qian, Y.H.; Li, C.H. Research on Feature Selection Method for Interval Sorting Decision. Chin. J. Manag. Sci. 2017, 25, 141-152. [CrossRef]

32. Wu, L.Y.; Yue, F.; Hu, K.; Wang, G. Innovation capability evaluation of universities based on combination of evidence grouping by interval number. Sci. Res. Manag. 2017, 4, 656-665. [CrossRef]

33. Zhang, J.G.; Song, X.W.; Yang, S. Buffer Sizing of a Critical Chain Project Based on the Entropy Method. Manag. Rev. 2017, 29, 211-219. [CrossRef]

34. Qian, Y.H.; Liang, J.Y.; Pedrycz, W.; Dang, C. Positive approximation: An accelerator for attribute reduction in rough set theory. Artif. Intell. 2010, 174, 597-618. [CrossRef]

35. Liang, J.Y.; Wang, F.; Qian, Y.H. A group incremental approach to feather selection applying rough set technique. IEEE Trans. Knowl. Date Eng. 2014, 26, 294-308. [CrossRef]

36. Xu, L.; Yao, X.L.; Li, Y.H. On the Risk Evaluation of Financing Guarantee Agencies based on Analytic Hierarchy Process. Econ. Manag. 2017, 31, 50-55. [CrossRef]

37. Song, P.; Liang, J.Y.; Qian, Y.H. A two-grade approach to ranking interval data. Knowl. Based Syst. 2012, 27, 234-244. [CrossRef] 
38. Billard, L.; Diday, E. From the Statistics of Data to the Statistics of Knowledge: Symbolic Data Analysis. J. Am. Stat. Assoc. 2003, 98, 470-487. [CrossRef]

39. Hu, T.; Jiao, Y.W. The efficiency evaluation of information networks based on interval-based cloud model. Syst. Eng. Theory Pract. 2017, 37, 478-485. [CrossRef]

40. Xu, X.H.; Wu, H.D. Approach for multi-attribute large group decision-making with linguistic preference information based on improved cloud model. J. Ind. Eng. Manag. 2018, 1, 117-125. [CrossRef]

41. Guo, Y.Q.; He, D.; Yao, Y. NMSI Model and Case Study in the Evolution of Innovation Ecosystem. Forum Sci. Technol. China 2017, 12, 25-31. [CrossRef]

42. Chen, X.D.; Chu, Q.Q. Research on Planning of Ecological Agricultural Park PV-Powered Based on Health-oriented Agriculture. J. Agric. Sci. Technol. 2017, 19, 45-51. [CrossRef]

43. Xue, J. Photovoltaic agriculture-New opportunity for photovoltaic applications in China. Renew. Sustain. Energy Rev. 2017, 73, 1-9. [CrossRef]

44. Fang, Y.D.; Huang, S.H.; Qing, S.X.; Shen, W.J.; Wei, D.; Yang, M.X.; Zheng, B.; Li, Y.J. Analysis of Development Status and Prospect of Photovoltaic Agriculture. J. Chang. Veg. 2015, 18, 35-40. [CrossRef]

(C) 2019 by the authors. Licensee MDPI, Basel, Switzerland. This article is an open access article distributed under the terms and conditions of the Creative Commons Attribution (CC BY) license (http://creativecommons.org/licenses/by/4.0/). 